\title{
A novel acoustic indoor localization system employing CDMA
}

\author{
Cem Sertatıl ${ }^{a}$, Mustafa A. Altınkaya ${ }^{a, *}$, Kosai Raoof ${ }^{b, c}$ \\ a Izmir Institute of Technology, Faculty of Engineering, Department of Electrical \&' Electronics Engineering, İzmir, Turkey \\ ${ }^{\mathrm{b}}$ Laboratoire GIPSA, Dept. DIS, Joseph Fourier University, France \\ c Laboratoire LAUM, ENSIM - University of Maine, Le Mans, France
}

\section{A R T I C L E I N F O}

\section{Article history:}

Available online 13 December 2011

\section{Keywords:}

Acoustic localization

DS-CDMA

Spread spectrum

Indoor localization

Time of arrival

\begin{abstract}
A B S T R A C T
Nowadays outdoor location systems have been used extensively in all fields of human life from military applications to daily life. However, these systems cannot operate in indoor applications. Hence, this paper considers a novel indoor location system that aims to locate an object within an accuracy of about $2 \mathrm{~cm}$ using ordinary and inexpensive off-the-shelf devices and that was designed and tested in an office room to evaluate its performance.

In order to compute the distance between the transducers (speakers) and object to be localized (microphone), time-of-arrival measurements of acoustic signals consisting of Binary Phase Shift Keying modulated Gold sequences are performed. This DS-CDMA scheme assures accurate distance measurements and provides immunity to noise and interference.

Two methods have been proposed for location estimation. The first method takes the average of four location estimates obtained by trilateration technique. In the second method, only a single robust position estimate is obtained using three distances while the least reliable fourth distance measurement is not taken into account.

The system's performance is evaluated at positions from two height levels using system parameters determined by preliminary experiments. The precision distributions in the work area and the precision versus accuracy plots depict the system performance. The proposed system provides location estimates of better than $2 \mathrm{~cm}$ accuracy with $99 \%$ precision.
\end{abstract}

(c) 2011 Elsevier Inc. All rights reserved.

\section{Introduction}

Location estimation is an important problem which has many applications in our everyday life. Although accurate outdoor location systems have been proposed, they cannot operate properly in indoor applications. Among these, Global Positioning System (GPS) [1] is an outstanding and effective outdoor location system that has worldwide coverage. Nevertheless, GPS cannot perform indoor localization. Therefore, specific location systems are needed for indoor localization applications. Especially in the last decade, the investigation on indoor localization has gained momentum, and the significance of indoor localization has been well established. There are many useful localization applications improving everyday life. Just a few common examples can be given as follows. Enterprises use indoor localization in order to localize their employees and their properties. In hospitals, doctors can be localized by an indoor location system, so they can reach their patients on time. In museums, visitors can be provided with information regarding

\footnotetext{
* Corresponding author.

E-mail addresses: sertatil@alumni.bilkent.edu.tr (C. Sertatıl), mustafaaltinkaya@iyte.edu.tr (M.A. Altınkaya), kosai.raoof@univ-lemans.fr (K. Raoof).
}

their current location or the system can show advertisements or location information to the user. Additionally, many location-aware applications become possible including access to nearby resources such as nearby printers or media-players.

In this work, we describe a novel indoor location system we developed using ordinary and inexpensive off-the-shelf devices with aim to locate entities in a room within $2 \mathrm{~cm}$ distance from its true position. We propose two methods of selecting the available distance measurements in order to use in trilateration defined in the next section. Robustly selecting three of the four measurements seems to be proposed for the first time. Our results also differ from the other results in literature in that we report all of the measurements whereas other works distinguish valid and invalid measurements.

The next section gives a brief overview of localization methods. Section 3 gives a historical account of location systems developed in the last two decades. Section 4 describes the features of the new location system, that is, the utilized equipment, the preparation of work area and the procedure followed in the localization. In Section 5, we explain the process of choosing some system parameters and test points in the work area. The last section contains the results of the experiments and comparisons with other location systems. 


\section{Methods of localization}

The traditional method of estimating a location is triangulation which is based on the geometric properties of triangles. Triangulation has two derivatives: angulation and lateration. Angulation technique corresponds to estimating the location of an object by using the angle of arrival information with respect to two and tree known locations for 2D and 3D location estimation, respectively. Lateration technique, on the other hand, corresponds to estimating the location of an object by using its distance from at least three and four reference points for $2 \mathrm{D}$ and 3D location estimation, respectively. Lateration with three reference points is called trilateration. If there are more measurements, it will be called multilateration. If more than necessary measurements are available, they can be combined in several ways. A typical way is minimizing a cost function in least square sense. In this work, we also tried rejecting the least reliable measurement in an effort to obtain a robust estimate.

There are again several ways of distance measurement for lateration techniques. Since distance is the product of the traveling time and the velocity, the traveling times of either an EM wave or a sound wave between the object to be located and some reference points are measured. The traveling time is called time of flight (ToF). If the time of departure at one end is known then the time of arrival (ToA) at the other end will give the ToF. In that case, the object's location will be estimated by regular tri(multi)lateration as the intersection of spheres whose centers and radii are the reference points and their distance to the object, respectively. If only time difference of arrival (TDoA) data, accordingly the difference of distances to references are known, the object's location will be at the intersection of hyperboloids. If ToA or TDoA data cannot be obtained reliably one can use the received signal strength (RSS), in other words the signal attenuation information corresponding to the traveled path. The RSS is a time-varying nonlinear function of the distance for indoor positioning which results in coarse-grained estimates. A more detailed record of methods for location estimation can be found in [2].

\section{A review of location systems}

Up to now, numerous investigations have been carried out about localization. Historically, the two localization examples for which well defined equipments are developed are radar and sonar. In radar, the distance of a far object at the instantaneous angle is determined by measuring the time needed by an electromagnetic (EM) wave to travel from the point of departure to the far object. In sonar, the traveling wave is an acoustic wave. Radar and sonar were greatly improved by the military requirements but they have been used also in many civilian applications. Nowadays, when we talk of location estimation, people would first think of many nonmilitary applications and global positioning system (GPS) based applications which is the foremost outdoor location system.

\subsection{Outdoor localization by the GPS}

The GPS was designed to meet both military and civilian requirements. 3D-localization of an object using the GPS is performed by measuring its distance from at least three GPS satellites. Actually, the measured quantity is the time required for signals to travel from the GPS satellites to the object to be located [3]. Since the EM waves travel with the speed of the light, a precise measurement of this duration is crucial. For public usage, the GPS system transmits a 1023-bits-long direct sequence code division multiple access (DS-CDMA) code, repeating thousand times a second which is called the coarse acquisition code (the C/A code). The code-phase civilian GPS trackers can synchronize to this code which is a $1 \mathrm{MHz}$ signal. During one chip (code bit) period the signal travels 300 meters which means that one chip inaccuracy corresponds to approximately to an error of 300 meters in estimating the distance. Fortunately, the synchronization error can be made 1 to 2 percent of 1 chip duration which makes the error in the distance estimate as low as 3 to 6 meters. More advanced signal processing techniques enables synchronization to the phase of the GPS signal carrying the C/A code which is a $1.57 \mathrm{GHz}$ stream and that results in much greater accuracy in distance estimation.

The GPS is unsuccessful in indoor localization because line-ofsight (LOS) transmission is not possible in most of the cases. So, other location systems are needed for indoor localization. Since the location estimation relies on distance measurement, the quality of the distance measurement determines the quality of the former one. A quantitative measure for the success of the estimation can be given by the terms accuracy and precision. The highest tolerated error for a measurement to be considered as successful will be referred to as target accuracy or shortly accuracy. In relation to that definition, the ratio of the measurements with errors equal to or less than the accuracy will be referred to as the precision of the measurements. Our motivation in this work is to perform inexpensive indoor localization with an accuracy of 2 centimeters which would make many location-aware applications possible.

\subsection{Indoor localization}

Indoor localization systems can be classified according to several aspects. One aspect is the type of the emitted signal which might be either an EM or an acoustic wave. According to the frequency of the EM wave used in the distance measurement, the technology utilized and the method of localization differ. EM wave-based location systems are infrared (IR) location systems, radio-frequency (RF) location systems using technologies such as RF identification (RFID), wireless local are network (WLAN), Bluetooth or ultra-wideband (UWB) and low frequency magnetic field location systems [4].

\subsubsection{EM wave-based}

An early indoor location system is Active Badge system [5] that is based on IR signals. It is used to localize people in an office environment. A badge worn by a person emits a unique code that is perceived from receptors at some fixed points. The master station processes data and displays it in a visual form. The system provides room-granularity accuracy, i.e. can determine in which room the person is. There are also some location systems which use camera arrays for sensing IR signals and achieve submillimeter accuracy, but these are very expensive systems. The IR location systems are influenced by interference from fluorescent light and sunlight. This interference problem can be solved by special filters for rejecting the interference [6] or noise canceling algorithms [7] which in turn increase the cost of the system.

RF-based indoor location systems are affected severely by multipath fading. However usually the already available infrastructure like the WLAN make them popular for localization. The WLANbased RADAR system $[8,9]$ measures the Signal Strength (SS) and signal-to-noise ratio (SNR) of the signals emitted from several known points with several known directions using three base stations and constructs fingerprinting data during off-line phase. In real-time phase, the user coordinates are estimated in two dimensions by matching the result obtained during the real-time phase with the fingerprints. The system estimates the user's location within $3 \mathrm{~m}$ accuracy and $50 \%$ precision that is $50 \%$ of the measurements are within $3 \mathrm{~m}$ proximity of the target. Another RF-based technology used for indoor location estimation is RadioFrequency Identification (RFID) technology. RFID tags reflect the RF 
signal they receive after modulating them with some added information. These tags are either passive or active. Passive RFID tags work without battery. However, their range is only 1-2 meters [2]. Active RFID tags are small transceivers and they support ranges as high as several tens of meters. One of the active RFID-based systems is LANDMARC (Location Identification based on Dynamic Active RFID Calibration) system [10] which uses a number of RFID readers and tags. The RFID readers have eight different power levels. Based on the received SS (RSS), the RFID reader determines which level the received power corresponds to. The process is implemented for each power level of each tag. Location of an object is estimated only with a low accuracy using the RSS data. Location systems using other RF-based technologies such as Bluetooth, cellular phone and cordless phone also use RSS for distance measurement and they achieve location estimates with several meters of accuracy [2,4]. There are deterministic and probabilistic methods of determining the location based on RSS data which are compared in a recent article [11]. UWB signals allow highest accuracy among RF-based location systems thanks to their ultrashort pulses of typically less than 1 ns duration which make it possible to distinguish the correct signal from its multipath replicas and using ToA measurement instead of RSS data. The achieved accuracies are $15-20 \mathrm{~cm}[2,4]$.

Since the IR- and RF-based location systems are either not finegrained or very costly, there is room for other more inexpensive and fine-grained location systems for which acoustic systems, in particular ultrasonic ones are good candidates.

\subsubsection{Acoustic wave-based}

In the BAT system, a small US transmitter, Bat, that is attached to a person emits a short unencoded pulse when base station transmits a radio message containing its identifier. The receivers placed at known positions record time of arrival (ToA) of any signal from the Bat. Consequently, the location of Bat is estimated by a central unit within $9 \mathrm{~cm}$ tolerance from its true position for 95 percent of the measurements using multilateration [12].

In order to solve the problem of determining the reference time, the Cricket system uses RF and US signals concurrently to measure the distance by calculating time difference of arrival (TDoA) of these signals $[13,14]$. In that system, beacons emit unmodulated US signals constructed by long enough radio messages. The location of a beacon is estimated by applying trilateration technique. It has room-granularity within an accuracy of a few inches. The difference of this system compared to its rivals is the firstly used decentralized (processing performed at the object's site) and privacy-oriented (others do not obtain knowledge of object's location) structure.

The Cricket Compass system [15] was improved from the Cricket system and it provides information about the orientation of the receiver. It uses V-shaped five US transducers to measure the phase differences in the received US signals.

The US location systems considered so far used unmodulated narrowband signals which resulted in low accuracy. Dolphin system $[16,17]$ is the first US location system which utilizes broadband signals in order to obtain fine-grained location estimates. It uses DS-CDMA method for simultaneous distance measurements as in GPS. The US ranging signal is modulated by 511-bits-long Gold code using BPSK. Two types of location systems have been proposed: the first one, polled and centralized and the second, privacy-oriented. In the polled and centralized system, the receivers are fixed to the ceiling and the location of emitter is estimated by applying multilateration technique using direct ToF measurement. It has approximately $2 \mathrm{~cm}$ accuracy. In the privacyoriented location system, the emitters are fixed to the ceiling while the receivers are roaming. This type of system can be synchronous or asynchronous. In the synchronous case, location of the receiver is estimated by conventional multilateration. On the other hand, in the asynchronous case location of the receiver is estimated by using direct sequence pseudorange measurements. Since the asynchronous transmission only provides relative measurements of ranges the measured distances are labeled with Pseudorange term. The system has $4.9 \mathrm{~cm}$ accuracy within $67 \%$ returned readings for synchronous case and $26.6 \mathrm{~cm}$ accuracy within 48\% returned readings for asynchronous case.

SmartLOCUS system [18] that is a self-assembling system consisting of ad-hoc networking nodes was proposed one year later It provides ease in adding new nodes. It uses RF and US signals concurrently to measure distances between nodes as Cricket system does. Additionally, RF signals are used to share the location data among nodes. The system has a distance accuracy of about $20 \mathrm{~cm}$ and the measurements are performed multiple times to get an accuracy figure about $1 \mathrm{~cm}$.

Another system [19] was improved from the Cricket system in 2007. In that system, the US pulses are encoded by thirteen-chipslong Barker code and modulated with BPSK to achieve greater accuracy in distance measurements. Besides that the update rate is improved by synchronizing the active beacons. The overall accuracy of the system is within $10 \mathrm{~cm}$.

Acoustic signals were used in 3D-LOCUS Local Positioning System (LPS) [20,21] that can determine the mobile target position with sub-centimeter accuracy. In 3D-LOCUS LPS, acoustic sound signals are modulated by 32-chips-long Golay code using BPSK. The system can perform in three different configurations: centralized, privacy-oriented and bidirectional. The results are obtained for the four different test conditions: Time Division Multiple Access (TDMA), Code Division Multiple Access (CDMA), TDMA with airflows and CDMA with airflows.

Some specifications of these indoor location systems are illustrated in Table 1 . These specifications are emitted signal, measurement technique, location estimation technique, structure, spread spectrum technique and performance of the system. The table also provides a quick comparison of the proposed location system to other existing systems.

Recently, extended versions of some interesting works demonstrated in the 2010 International Conference on Indoor Positioning and Indoor Navigation (IPIN) are published. There are some interesting localization studies using acoustic signals among them. In [22] a DS-CDMA-based US system which is Doppler shift tolerant, is proposed in order to determine the location of moving entities. In [23] a Kasami code-based US system's performance was given as influenced by the properties of the transducer, multipath propagation and receiver movement. [24] uses US chirp signals and individual PN code-based wireless sensor network whereas [25] uses US signals and a variant of the well-known direction finding algorithm MUSIC. In [26], a system with a single US receiver is given which estimates both the position and the velocity of an entity.

The measurement method in our system is similar to the Dolphin system. But differently, we utilize trilateration instead of multilateration. We propose two methods of selecting the available distance measurements in order to use in trilateration. In the second method, selecting three of the four measurements makes it possible to eliminate the most unreliable estimates which leads to more robust location estimates. In contrast to the location systems mentioned above, we report all of the measurements whereas other works distinguish valid and invalid measurements. Now, let us continue with the details of the proposed system.

\section{The new location system}

In this paper, we propose a new indoor location system that is based on acoustic transducers. Acoustic signals are used to locate 
Table 1

Specifications of indoor location systems.

\begin{tabular}{|c|c|c|c|c|c|c|}
\hline & Emitted signal & $\begin{array}{l}\text { Measurement } \\
\text { technique }\end{array}$ & $\begin{array}{l}\text { Location estimation } \\
\text { technique }\end{array}$ & Structure & $\begin{array}{l}\text { Spread spectrum } \\
\text { technique }\end{array}$ & Accuracy and precision \\
\hline Active Badge (1992) & IR & Connectivity & Proximity & Centralized & - & $\begin{array}{l}\text { Coarse-grained } \\
\text { Room-granularity }\end{array}$ \\
\hline BAT (1997) & US & ToA & Multilateration & Centralized & - & $\begin{array}{l}\text { Fine-grained } \\
9 \mathrm{~cm}(95 \%)\end{array}$ \\
\hline RADAR (2000) & $\mathrm{RF}$ & RSSI & $\begin{array}{l}\text { Fingerprinting or } \\
\text { Triangulation }\end{array}$ & Centralized & - & $\begin{array}{l}3 \mathrm{~m}(50 \%) \\
\text { Room-granularity }\end{array}$ \\
\hline Cricket (2000) & RF and US & $\begin{array}{l}\text { TDoA or } \\
\text { Connectivity }\end{array}$ & $\begin{array}{l}\text { Trilateration or } \\
\text { Proximity }\end{array}$ & $\begin{array}{l}\text { Decentralized } \\
\text { Privacy-oriented }\end{array}$ & - & $\begin{array}{l}\text { A few inches } \\
\text { Room-granularity }\end{array}$ \\
\hline Cricket Compass (2001) & RF and US & $\begin{array}{l}\text { Phase } \\
\text { difference }\end{array}$ & - & $\begin{array}{l}\text { Decentralized } \\
\text { Privacy-oriented }\end{array}$ & - & $\begin{array}{l}3^{\circ} \pm 30^{\circ} \\
5^{\circ} \pm 40^{\circ}\end{array}$ \\
\hline \multirow[t]{3}{*}{ Dolphin (2002) } & US & ToA & Multilateration & $\begin{array}{l}\text { Polled and } \\
\text { centralized }\end{array}$ & $\begin{array}{l}\text { DS-CDMA } \\
511 \text { Gold } \\
\text { Code BPSK }\end{array}$ & $\begin{array}{l}2.1 \mathrm{~cm} \text { accuracy } \\
\text { with } 95 \% \text { confidence } \\
\text { ( } 75 \% \text { valid readings) }\end{array}$ \\
\hline & & & & Privacy-oriented & & $\begin{array}{l}\text { Synchronous } \\
4.9 \mathrm{~cm} \text { accuracy } \\
\text { with } 95 \% \text { confidence } \\
\text { ( } 67 \% \text { valid readings) }\end{array}$ \\
\hline & & & & & & $\begin{array}{l}\text { Asynchronous } \\
26.6 \mathrm{~cm} \text { accuracy } \\
\text { with } 95 \% \text { confidence } \\
\text { ( } 48 \% \text { valid readings) }\end{array}$ \\
\hline SmartLOCUS (2003) & RF and US & TDoA & - & $\begin{array}{l}\text { Decentralized } \\
\text { self-assembling }\end{array}$ & - & $\begin{array}{l}20 \mathrm{~cm} \text { distance } \\
\text { accuracy }\end{array}$ \\
\hline LANDMARC (2004) & RF (RFID) & RSSI & $\begin{array}{l}\text { Proximity or } \\
\text { Fingerprinting }\end{array}$ & Decentralized & - & Coarse-grained \\
\hline Piontek et al. (2007) & RF and US & TDoA & Multilateration & $\begin{array}{l}\text { Decentralized } \\
\text { Privacy-oriented }\end{array}$ & $\begin{array}{l}\text { DS-CDMA } \\
13 \text { Barker } \\
\text { Code BPSK }\end{array}$ & $10 \mathrm{~cm}$ \\
\hline \multirow[t]{2}{*}{ 3D-LOCUS (2007) } & Acoustic & ToA & Trilateration & Centralized & $\begin{array}{l}\text { DS-CDMA } \\
32 \text { Golay } \\
\text { Code BPSK }\end{array}$ & $\begin{array}{l}12.6 \mathrm{~mm} \text { accuracy } \\
\text { with } 95 \% \text { confidence } \\
(96.2 \% \text { valid readings })\end{array}$ \\
\hline & & & & Privacy-oriented & & $\begin{array}{l}13.5 \mathrm{~mm} \text { accuracy } \\
\text { with } 95 \% \text { confidence } \\
\text { (94.7\% valid readings) }\end{array}$ \\
\hline Proposed system (2010) & Acoustic & ToA & Trilateration & Centralized & $\begin{array}{l}\text { DS-CDMA } \\
511 \text { Gold } \\
\text { Code BPSK }\end{array}$ & $\begin{array}{l}2 \mathrm{~cm} \text { accuracy } \\
\text { with 99\% precision }\end{array}$ \\
\hline
\end{tabular}

several microphones physically in 3D coordinate system. A centralized unit, PC, performs the computations and controls the system. The location system can locate the microphones attached to the entities simultaneously using DS-CDMA signals with accuracy order of centimeters. The CDMA signals emitted from the speakers are BPSK modulated Gold code sequences. Good auto-correlation properties of these codes are used in obtaining accurate time measurements whereas their good cross-correlation property provides high processing gains and in turn simultaneous measurement possibility of all the required distances without making a significant disturbance to each other. The distances are estimated by using ToA measurement technique and the locations are estimated by using trilateration.

Acoustic signals are preferred due to their low velocity that makes an accurate localization possible with available inexpensive devices. A centralized unit is used for ease of computation. The centralized location system can work with simple devices since the nodes do not need to make any computations. DS-CDMA provides simultaneous signal emission from all of the speakers and robustness in signal-to-noise and interference ratio. Gold code sequences are preferred since a large number of codes can be generated with good auto-correlation and cross-correlation properties. Orthogonal codes are not proper candidates for this system since times of arrivals of the signal to the microphone are different. Hence orthogonal codes cannot be separated from each other. The small set of Kasami codes comprise only the codes generated from even number of shift registers, so they are not suitable, either. On the other hand, m-sequences and Barker code sequence do not have many numbers of codes. Trilateration technique was preferred rather than multilateration for ease in computation. Trilateration requires synchronization between transmitters and receivers, while multilateration requires only synchronization between receivers to measure the pseudoranges. Although speakers and the receivers are synchronized in the proposed system, some delays occur in transmission. Hence an additional microphone with known location is used in order to find the delays.

\subsection{Equipment}

Cost is one of the most important issues for manufacturers and customers. Inexpensive products with similar capability are preferred to expensive ones. Therefore, ordinary and inexpensive devices were used in the location system we implemented. While most of them were off-the-shelf devices, some of them were produced and assembled by us in order to reduce the implementation cost. But they could also have been bought for a small price.

As shown in Fig. 1, the system hardware consists of a central unit, an external sound card, a car amplifier, four tweeters, a digital thermometer and at least two microphones. An ordinary PC that is capable to run MATLAB, was used as the central unit. On the other hand, an external sound card with four uniform output and two uniform input channels was used to process the acoustic 


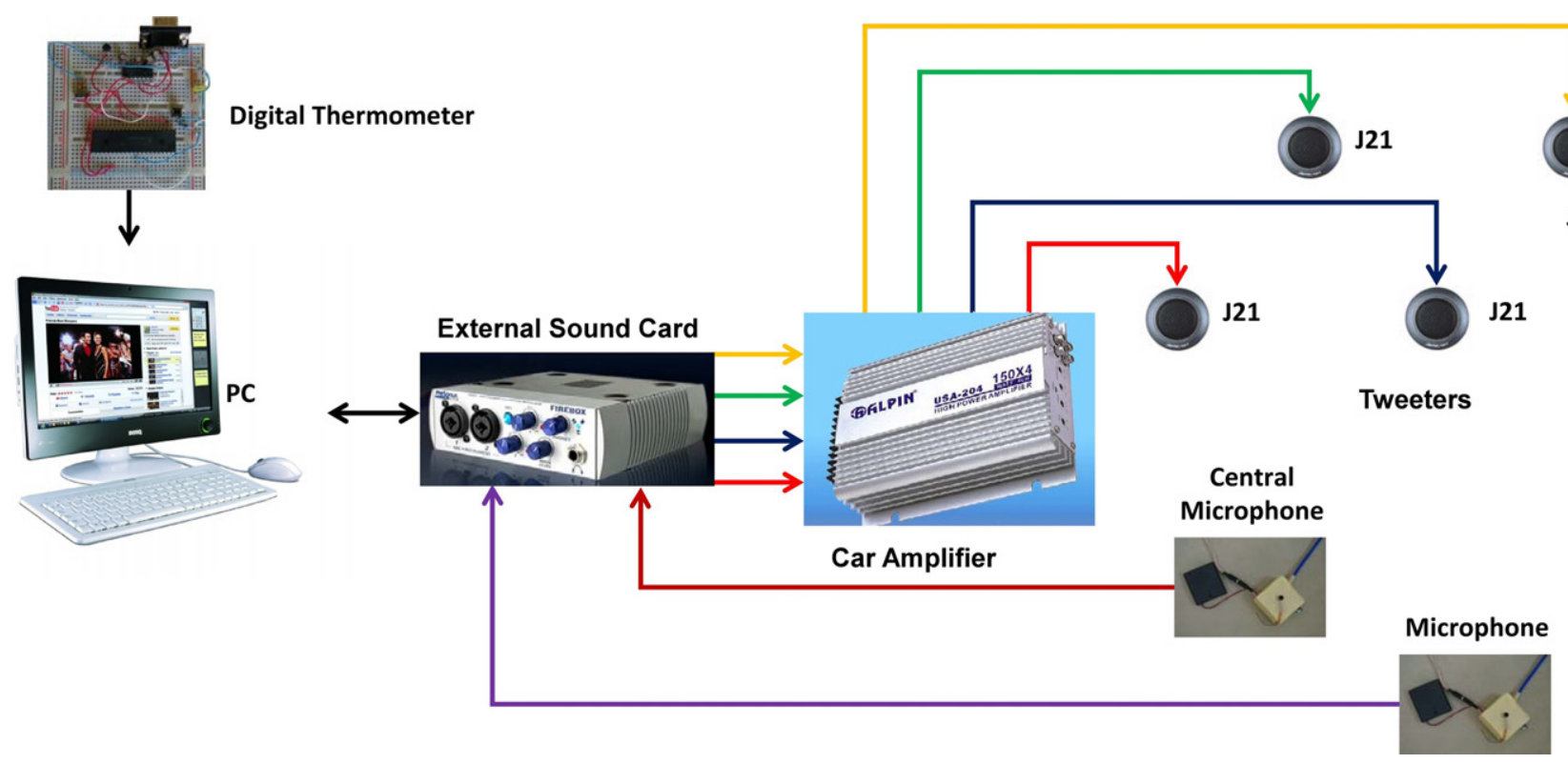

Fig. 1. Connection diagram of location system.

signals. This device can sample analog data with frequency up to $96 \mathrm{kHz}$. The four distinct analog sound signals which are produced by the PC are amplified by the car amplifier before they are emitted from the speakers. The acoustic system composed by the external sound card and the car amplifier provides four simultaneous distinct channels for broadcasting and two simultaneous distinct channels for recording the incoming data from the two microphones. The chosen tweeters are inexpensive off-the-shelf devices whereas the two microphones were produced and assembled in order to reduce the cost. In addition to these devices, a digital thermometer is also utilized in order to measure the ambient temperature which is essential for calculating the sound velocity. On the software side, data acquisition between the PC and the external sound card is performed using the related toolbox of MATLAB.

\subsection{Preparation of work area}

After the devices were provided, an office in the Department of Electrical \& Electronics Engineering at İzmir Institute of Technology was allocated. In order to measure the performance of the system free from the specific surroundings in a certain room

- the work area was encircled with curtain,

- the ceiling was covered with a material of low reflectivity,

- the floor was covered with carpet,

as shown in Fig. 2. These arrangements assured reducing the effects of exterior influences and multipath disturbances due to the reflections.

The experimental set-up was built as shown in Fig. 3. The dimensions of the area are $1.5 \mathrm{~m}$ in width, $2 \mathrm{~m}$ in length and $3 \mathrm{~m}$ in height. The speakers were placed at the corners of the ceiling as shown in the figure. The projection of tweeter 1 at the floor is set to be the origin in the $3 \mathrm{D}$ coordinate system. Tweeter- 1 , tweeter-2, tweeter- 3 and tweeter- 4 were located at the $(x, y, z)$ coordinates $(0,0,3),(1.5,0,3),(0,2,3)$ and $(1.5,2,3)$, respectively while the central microphone was placed at the center of the floor, $(0.75,1,0)$, due to the convenience of its being at equal distance to each tweeter. Another microphone was also used as the object whose location is to be determined. Actually, the system can locate

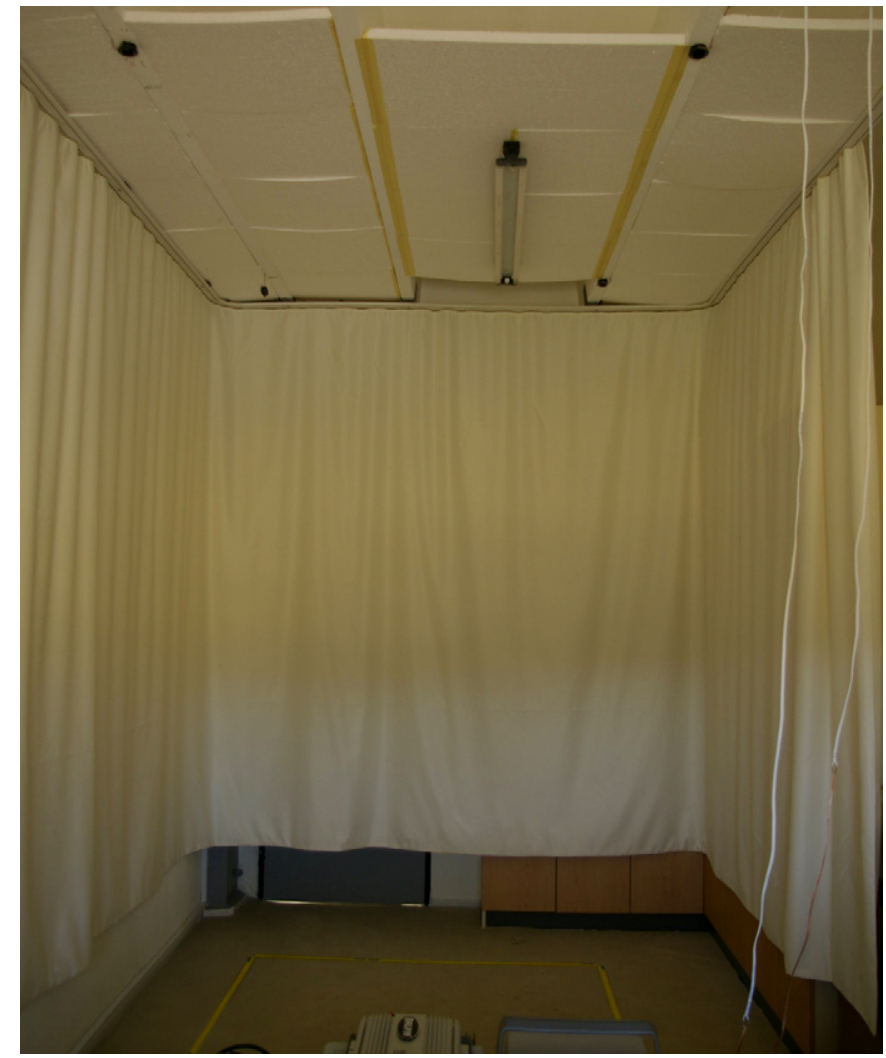

Fig. 2. Photo of the work area.

more than one microphone simultaneously, once they are connected to the system. However, in this implementation only one microphone was used for locating its position.

\subsection{Procedure of localization}

In the proposed location system, localization is performed by following the three main operation blocks shown in Fig. 4 in order. Let us explain these operation blocks in detail. 


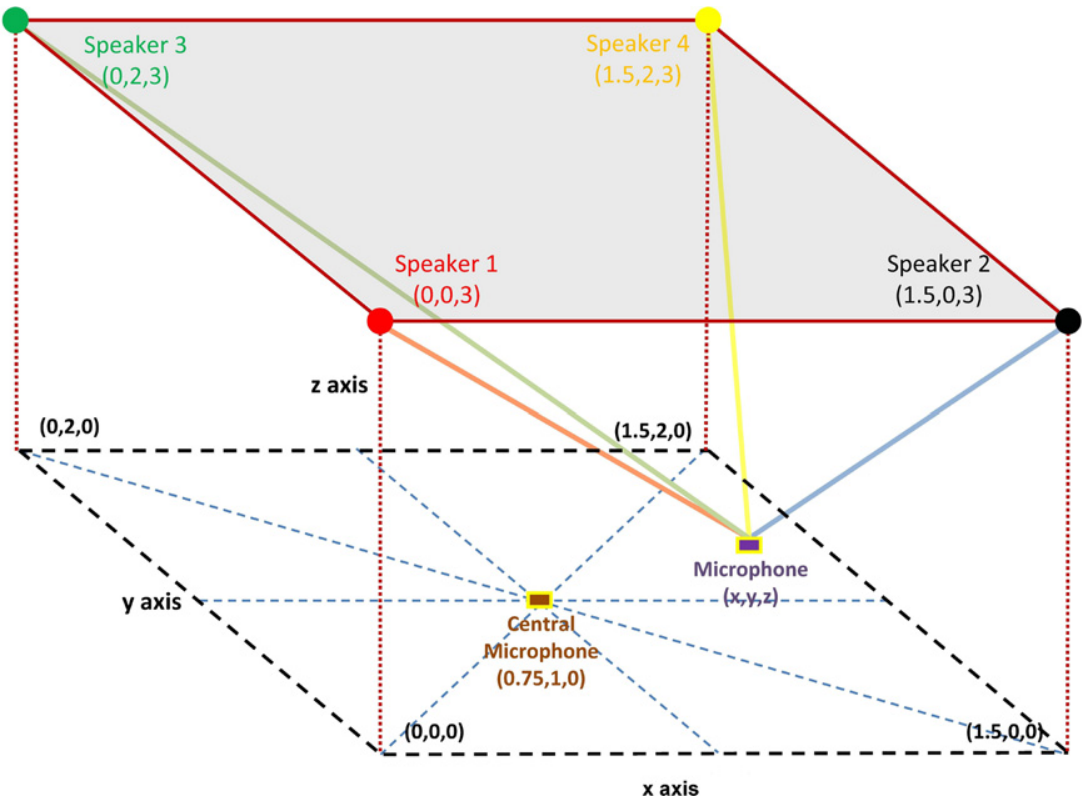

Fig. 3. The set-up at the office.

PRE-CALCULATIONS

-Determine parameters
$\cdot$ Measure temperature \&
calculate $\mathrm{v}_{\text {sound }}$
$\cdot$ Generate PN-codes
-Create modulated data signal

DISTANCE MEASUREMENT

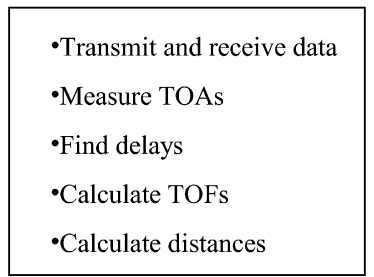

Fig. 4. Location estimation procedure.

\subsubsection{Precalculations}

As a first step the values for some system parameters such as the code-length, the sampling frequency, the carrier frequency and the chip frequency are set. The empirical determination of the best choices is explained in Section 5.1.

For calculating distances by means of acoustic signals, the velocity of acoustic signals must be known. There are two ways to determine the velocity. As a first alternative, the velocity of the sound can be determined from its nominal value at a certain temperature since it is a function of temperature. The formula

$v_{\text {sound }}=331.3 \sqrt{1+\frac{T}{273.15}}$

uses the velocity of the sound at $0{ }^{\circ} \mathrm{C}$ as a reference in order to find its value $v_{\text {sound }}$ in $\mathrm{m} / \mathrm{s}$ at a temperature $T$ in ${ }^{\circ} \mathrm{C}$. The other way to determine the velocity is to measure the duration that the signal needs for traveling a known distance. In our system, we calculated the velocity using (1). We measured the ambient temperature with a digital thermometer which we produced and assembled. Actually, humidity and wind are also effective on velocity of sound. However, the influence of the humidity is negligible compared to the one of the temperature and the wind is not considered in our work. The effect of indoor airflows due to air-conditioning was investigated in $[20,21]$. In order to reduce the influence of wind, [27] suggested estimating the sound velocity continuously by measuring the travel time for a known distance in proximity and correcting the other measurements accordingly.

The next step is to produce CDMA signals. For this a Gold code matrix was generated according to a definite number of shift registers. Four distinct Gold codes were selected randomly. The codes must be distinct since each code is assigned to a speaker. The four codes that have good auto-correlation and cross-correlation properties could have been selected, but in this implementation it is preferred to select the codes randomly in order not to lose generality of our set-up to determine the location of multiple entities distributed in a larger area for which an accordingly large grid of sensors with the same sensor-density can be deployed. After selecting the four distinct Gold codes, these codes were modulated with BPSK to obtain the acoustic signals.

\subsubsection{Distance measurement}

The four distinct data signals each identifying the speaker it originates with a unique Gold code, were amplified by a car amplifier and transmitted simultaneously from the speakers. Two microphones were used for receiving the aggregate data signal. One microphone was located at a fixed location, at the center of the floor, and was used for determining the delays for each speaker. For this purpose, the difference between the computed time and the measured time had to be calculated. The definitions of various parameters in the measurement process are demonstrated in Fig. 5. First, the distance between a speaker and the central microphone, $d_{\text {cal }}$, was calculated by using basic geometry. Then the number of sampling periods, $L$, that is needed for an acoustic signal to travel from that speaker to the central microphone was computed as

$L=\frac{d_{\text {cal }} f_{\text {sampling }}}{v_{\text {sound }}}$

where $f_{\text {sampling }}$ is the sampling frequency.

After computing $L$, the aggregate data signal that was received by the central microphone was correlated with the data signal sent 


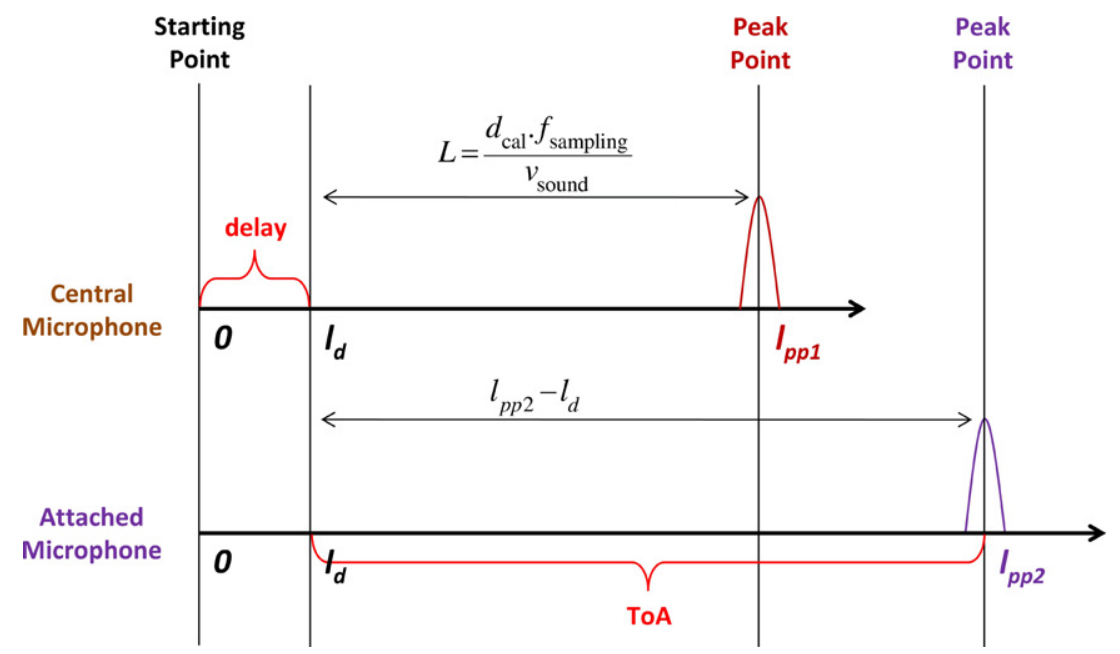

Fig. 5. Finding delay and distance.

from that speaker. Then the peak correlation value for the correlation sequence was selected. The instance of the peak correlation in terms of sampling period, $l_{p p 1}$, was found. Eventually, subtracting $L$ from $l_{p p 1}$ gave the delay, $l_{d}$, for that speaker.

After finding the delay for that speaker, ToA measurement technique was used for finding the distances. The process for finding distances is similar to the process for finding delays. The signal acquired from the other microphone attached to an entity was correlated with the original data signal. The correlations gave a high peak thanks to the good correlation properties of Gold codes. The peak correlation value for the correlation sequence was selected. The time of the new peak point, $l_{p p 2}$, was found as in the previous process. The measured distance $d_{m}$ found as

$d_{m}=\frac{\left(l_{p p 2}-l_{d}\right) v_{\text {sound }}}{f_{\text {sampling }}}$

gave the distance from the speaker to the entity. This process was repeated for each speaker to find the four distinct distances.

\subsubsection{Estimating the location}

As a last step, trilateration method was applied in order to estimate the location of the microphone attached to the entity. The four distances were computed in the previous step. Indeed, three distances are sufficient to locate an object by using trilateration technique and since three distances out of four can be chosen in four ways, four different location estimates can be computed. Consequently, two methods were proposed.

Method I uses three of the four measured distances to obtain a location estimate. There will be 4 combinations of 3 ToA values to obtain 4 different location estimates which we denote by coordinates $\left\{P_{i}\left(x_{i}, y_{i}, z_{i}\right) ; i=1,2,3,4\right\}$. The location estimate of this method is simply the center point of the four estimates given by

$P\left(x_{p}, y_{p}, z_{p}\right)=P\left(\frac{1}{4} \sum_{i=1}^{4} x_{i}, \frac{1}{4} \sum_{i=1}^{4} y_{i}, \frac{1}{4} \sum_{i=1}^{4} z_{i}\right)$.

On the other hand, Method II uses only three measured distances. In order to select three distance measurements out of four, the least reliable distance measurement which corresponds to the minimum value of the peak correlations is eliminated. Then, the location of the microphone in terms of $x_{p}, y_{p}, z_{p}$ is computed by using trilateration.

It can be said that Method I considers all of the four distance measurements evenly reliable and gives them equal weight in obtaining the location estimate. On the contrary, Method II eliminates the least reliable distance measurement corresponding to the smallest correlation peak. So, one might assert that Method II will give a more robust localization estimate when one of the measurements is significantly worse compared to others. If the measurements are of comparable quality, Method I is expected to be more successful because it uses more information compared to Method II.

\section{Determination of the system parameters}

\subsection{Choosing the free parameters}

Considering the described procedure of localization, there are some free parameters to be selected. We tried to optimize them empirically to achieve our design goal of $2 \mathrm{~cm}$ accuracy with satisfactory results within tolerance. The investigated system parameters are:

(i) length of the Gold code sequence,

(ii) sampling frequency,

(iii) ratio of carrier frequency to chip frequency,

(iv) carrier frequency.

In all of these simulations the performance criterion is the percentage of realizations within a tolerance where this tolerance changing from $5 \mathrm{~mm}$ to $3 \mathrm{~cm}$ is a parameter. The nominal values for the system parameters are

- carrier frequency: $f_{\text {carrier }}=15 \mathrm{kHz}$,

- chip frequency: $f_{\text {chip }}=15 \mathrm{kHz}$,

- sampling frequency: $f_{\text {sampling }}=96 \mathrm{kHz}$,

in these simulations and Method II was applied for estimating the location.

Fig. 6 depicts the performance of the system with respect to the change in the length of the Gold code sequence. For all of the tolerated levels of position error a performance threshold is seen at the codeword length of 127 bits. Such a threshold is expected since the cross-correlations of the codewords decrease as their lengths increase. At that threshold more than $97 \%$ of readings are within $2 \mathrm{~cm}$ proximity of the true location. It is seen that 127-bits-long and 256-bits-long Gold code sequences exhibit substantially close results among them. This behavior is due to the common normalized peak correlation values of these codes. If this performance is not satisfactory one should use 511-bits-long Gold codes. Hence, according to these simulation results 127-bits-long and 511-bitslong Gold sequences appear as possible choices, the first one and 


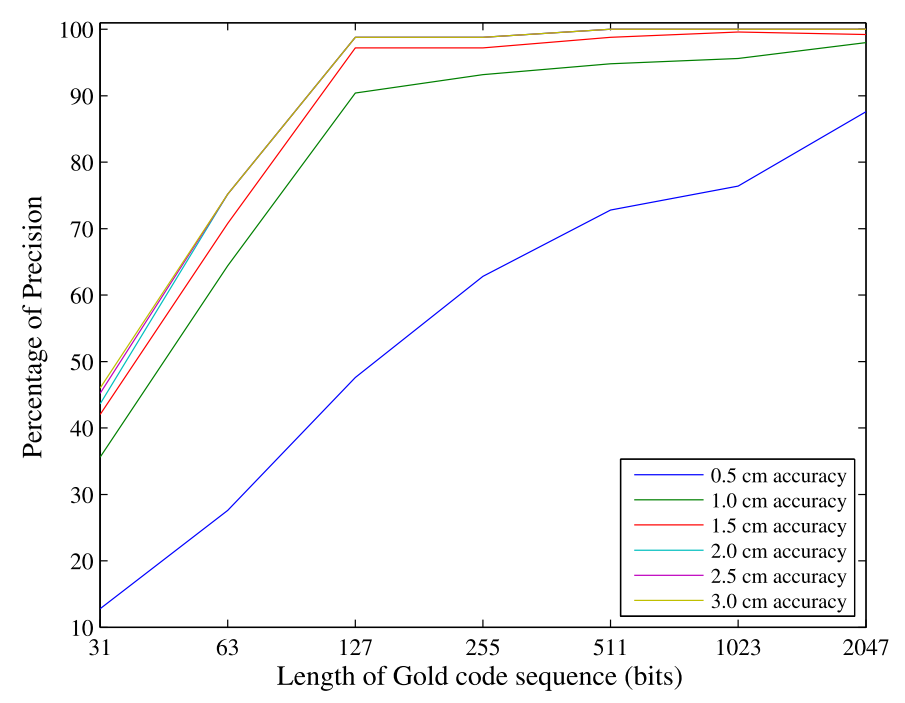

Fig. 6. Performance of the system against the length of Gold code sequence.

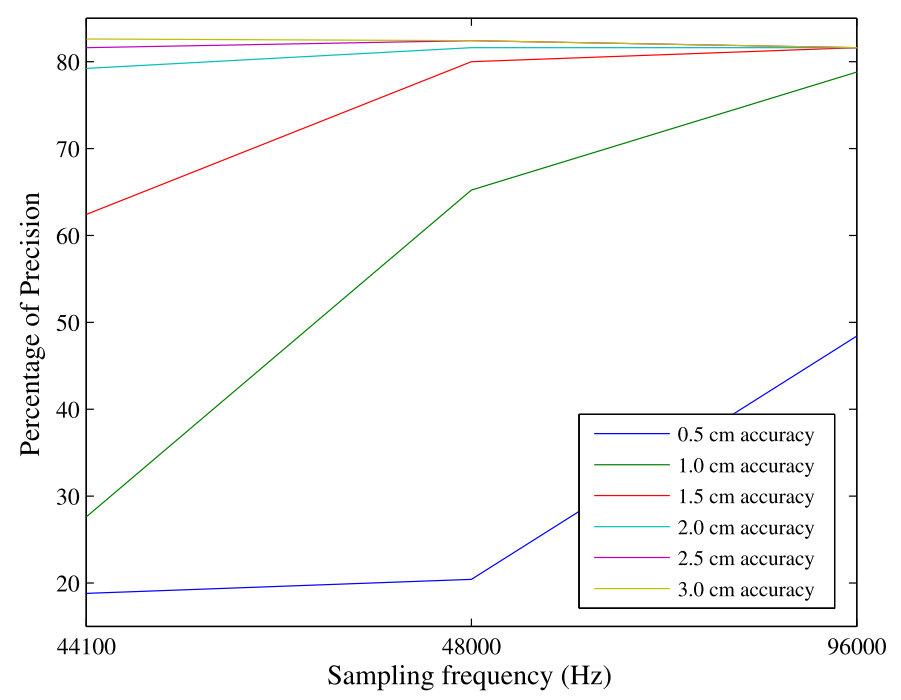

Fig. 7. Performance of the system against the sampling frequency.

the second one being more precise and less computational time demanding, respectively.

Secondly, the performance of the system as a function of the sampling frequency where the choices are $44100 \mathrm{~Hz}, 48000 \mathrm{~Hz}$ and $96000 \mathrm{~Hz}$, was evaluated. The length of the Gold code sequence was selected as 127 bits. Method I was applied for location estimation. Fig. 7 depicts that for any level of tolerated position error the performance increases as the sampling frequency is increased. The reason is that higher sampling frequency corresponds to finer resolution in ToA and consequently in distance estimates which demonstrates itself with better time correlation properties. Therefore $96000 \mathrm{~Hz}$ was selected as the sampling frequency.

Fig. 8 shows the performance of the system as a function of the ratio of carrier frequency to chip frequency. Carrier frequency was selected as $15000 \mathrm{~Hz}$ and chip frequency was varied accordingly. The figure depicts that ratio 1 and ratio 1.25 gave better results compared to others. For computational convenience ratio 1, which means carrier frequency and chip frequency take the same value, was selected.

Next, the performance of the system was evaluated as a function of the carrier frequency. Carrier and chip frequencies took the same values from $5 \mathrm{kHz}$ to $22 \mathrm{kHz}$. Fig. 9 depicts that the highest number of realizations within tolerance are obtained for a

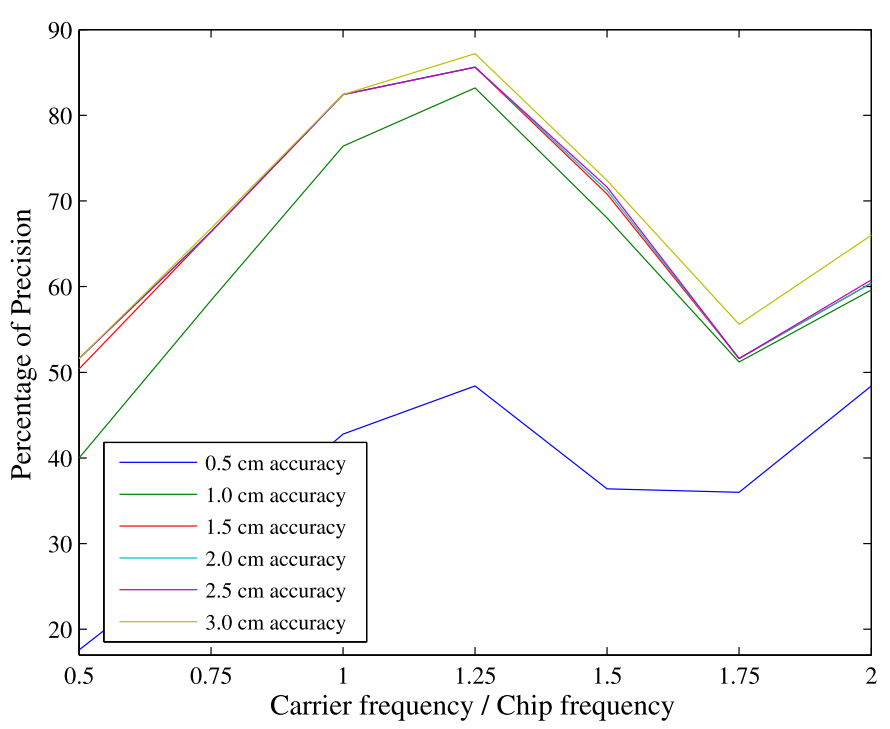

Fig. 8. Performance of the system against the ratio of carrier frequency to chip frequency.

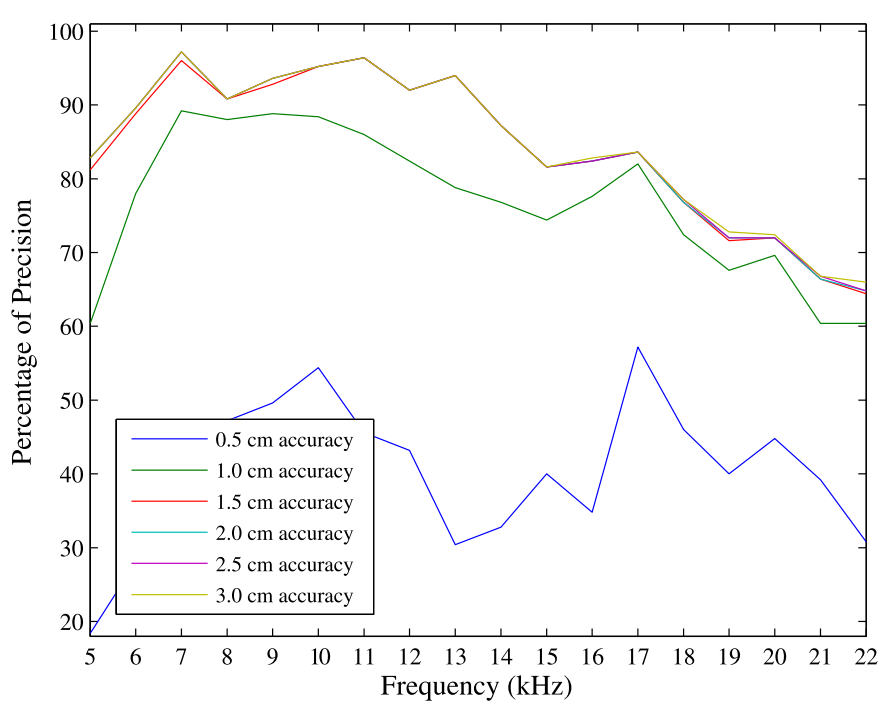

Fig. 9. Performance of the system against the frequency.

sound frequency of $7000 \mathrm{~Hz}$ and the performance gets worse with increasing frequency. In order to attain a synchronization which corresponds to a fraction of the chip period, many samples per chip are needed which corresponds to a considerably smaller chip rate compared to the sampling frequency. That is why a downward trend in the precision results accompanies the increase in the carrier frequency beyond approximately $7000 \mathrm{~Hz}$. There is an exceptional performance characteristic for an accuracy target of $5 \mathrm{~mm}$ but we do not consider this curve since that high level accuracy seems to be beyond the capabilities of the designed system shown by the fact that the percentage of realizations within $5 \mathrm{~mm}$ position error do not exceed approximately 55\% for any acoustic frequency.

In addition to these four parameters, the methods for location estimation were also examined. According to empirical results, Method I that is thrusting evenly to any of the four ToA measurements is more advantageous for a codeword length of 511 chips whereas Method II becomes more advantageous in the case of 127-chips-long Gold codes. In the later case, it was observed that one of the distances, usually the longest one, cannot be mea- 


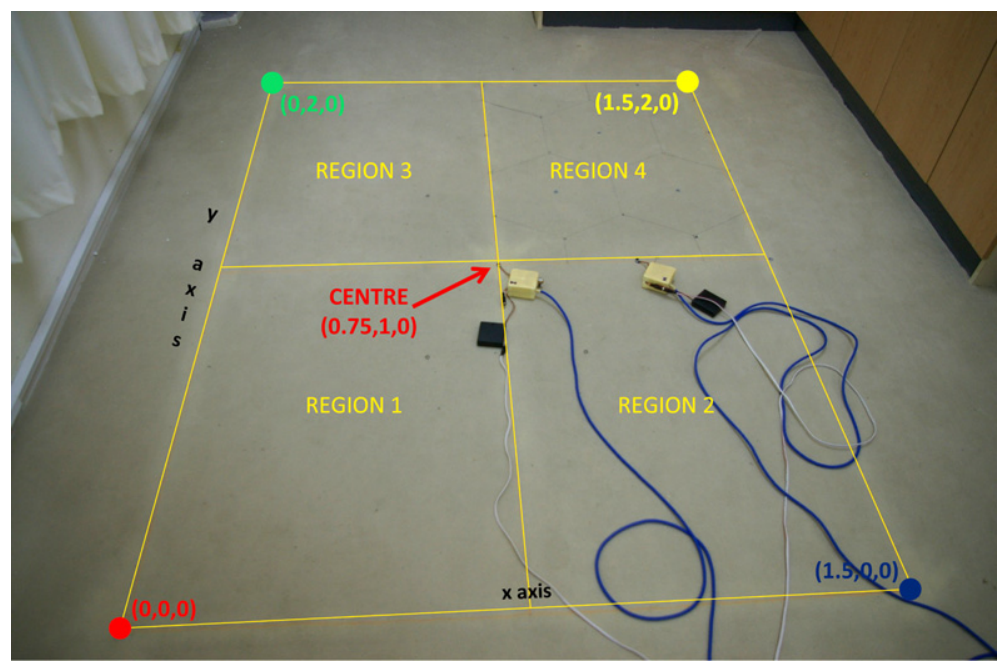

Fig. 10. The regions in the work area.

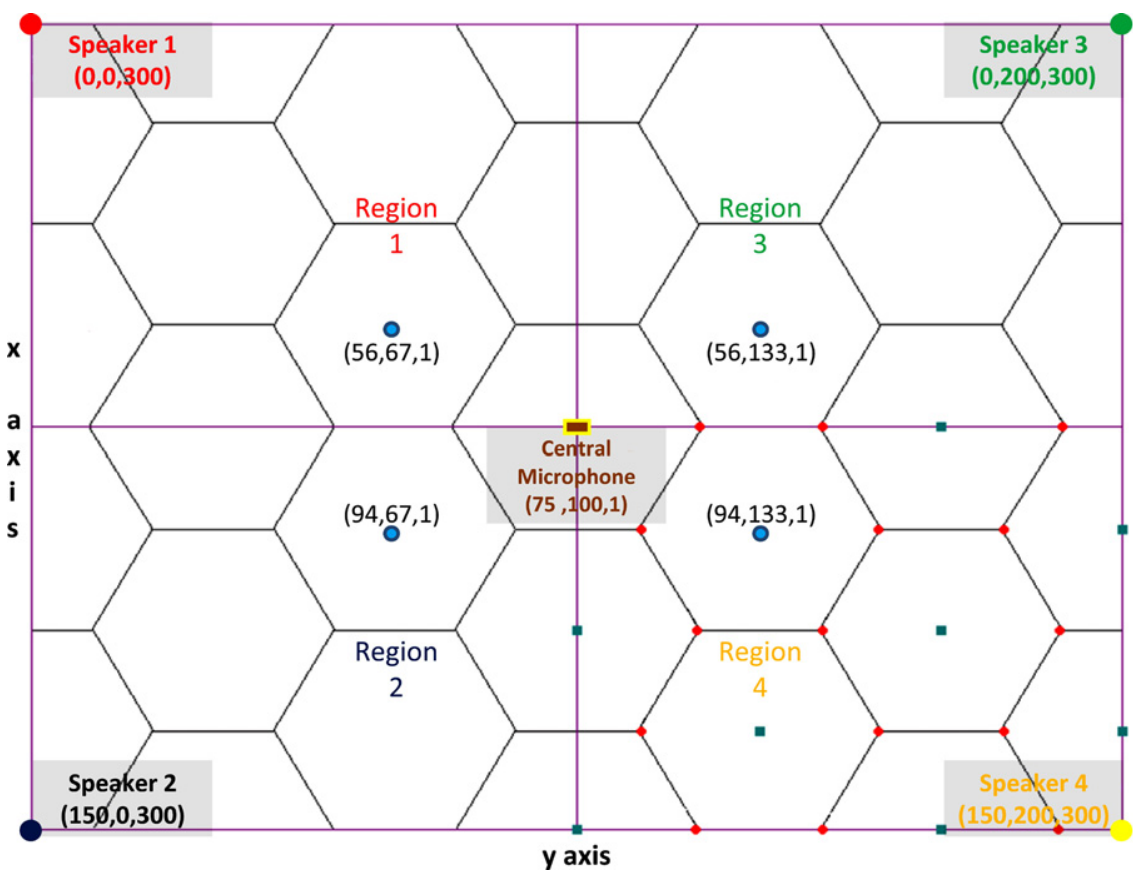

Fig. 11. The selected equivalent points taken from different regions.

sured accurately enough. Therefore applying Method II instead of Method I robustifies the location estimate. as:

After these preliminary tests the system variables were selected

- $96000 \mathrm{~Hz}$ for sampling frequency,

- 511 bits for length of Gold code sequence,

- $7000 \mathrm{~Hz}$ for carrier and chip frequencies,

- Method I for location estimation method.

\subsection{Choosing the test points}

After selecting the system variables, the test points were defined in the work area. To reduce the number of the test points, the work area was divided into four equivalent rectangular regions as shown in Fig. 10.

In order to validate that the defined regions are equivalent regarding the performance of the proposed location system, four presumably equivalent points were selected from the four regions. The selected points are illustrated in Fig. 11 that depicts the work area with a ninety degrees clockwise rotation with respect to Fig. 10.

In Fig. 12, percentage of realizations within tolerance were plotted as a function of the desired accuracy for four equivalent points taken from four rectangular subregions of the work area. The highest accuracy difference which is approximately $4 \mathrm{~mm}$, occurs between the points from Regions 3 and 4 for $80 \%$ realizations within accuracy. Also for an accuracy tolerance of $15 \mathrm{~mm}$ at all of the equivalent points, all of the readings have smaller position errors than the tolerance. Consequently, we decided to select one of the regions for our experiments without sacrificing the generality of the results and selected Region 4 for the detailed experimental investigation.

A group of test points were selected at the ground level that is 3 meters below the speakers and another group of test points were selected at a level which is 1 meter above the ground level. Fig. 13 illustrates the ground level test points. The ground level test points were taken as the centers of hexagons as shown in the figure. 


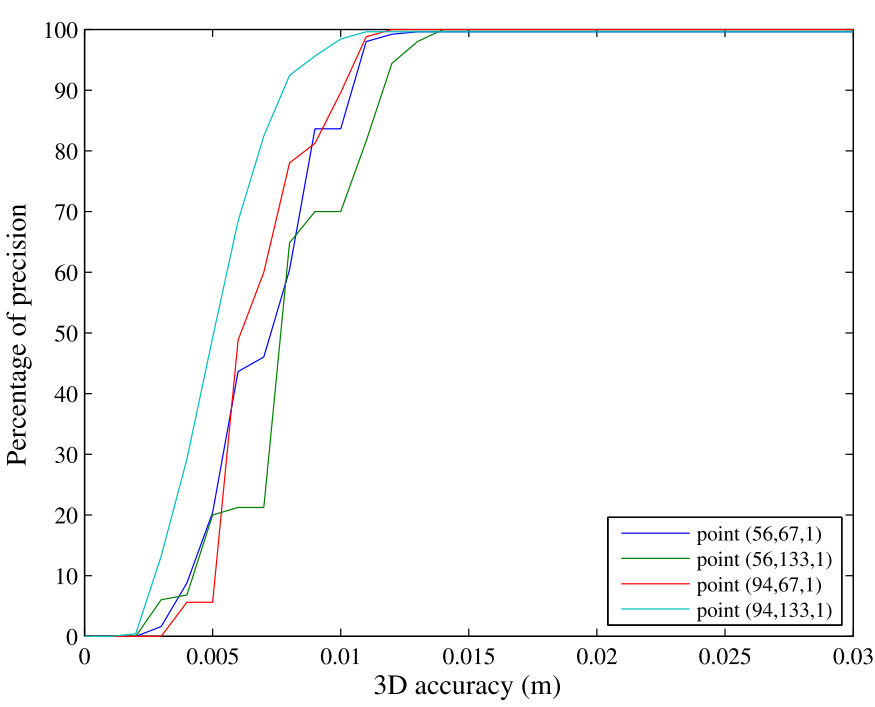

Fig. 12. Precision versus accuracy of the four equivalent points.

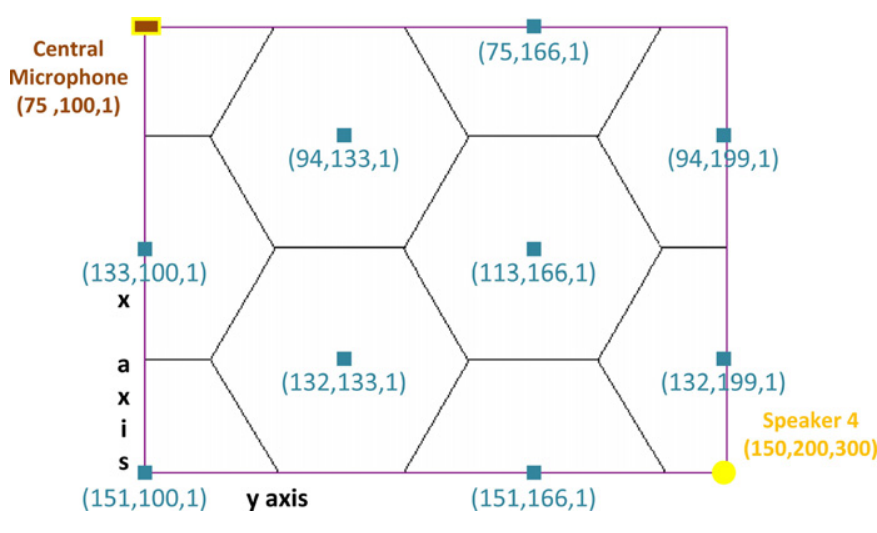

Fig. 13. Ground level test points.

\section{Results and discussion}

The precision results corresponding to several accuracy levels obtained from ground level test points were collected in Table 2.
Table 2

Percentages of precision at ground floor.

\begin{tabular}{lllcccc}
\hline Points & Accuracy & \multicolumn{5}{c}{} \\
\cline { 2 - 7 } & $0.005 \mathrm{~m}$ & $0.01 \mathrm{~m}$ & $0.015 \mathrm{~m}$ & $0.02 \mathrm{~m}$ & $0.025 \mathrm{~m}$ & $0.03 \mathrm{~m}$ \\
\hline $113,100,1$ & 37.6 & 97.6 & 99.6 & 99.6 & 99.6 & 99.6 \\
$151,100,1$ & 17.2 & 91.2 & 100 & 100 & 100 & 100 \\
$94,133,1$ & 49.2 & 98.4 & 99.6 & 99.6 & 99.6 & 99.6 \\
$132,133,1$ & 51.2 & 99.2 & 100 & 100 & 100 & 100 \\
$75,166,1$ & 16 & 92.4 & 99.6 & 99.6 & 99.6 & 99.6 \\
$113,166,1$ & 13.6 & 80 & 98.8 & 98.8 & 98.8 & 98.8 \\
$151,166,1$ & 0 & 64.8 & 99.2 & 99.2 & 99.2 & 99.2 \\
$94,199,1$ & 0 & 20.4 & 79.6 & 98.8 & 99.6 & 99.6 \\
$132,199,1$ & 0 & 47.2 & 93.2 & 100 & 100 & 100 \\
\hline
\end{tabular}

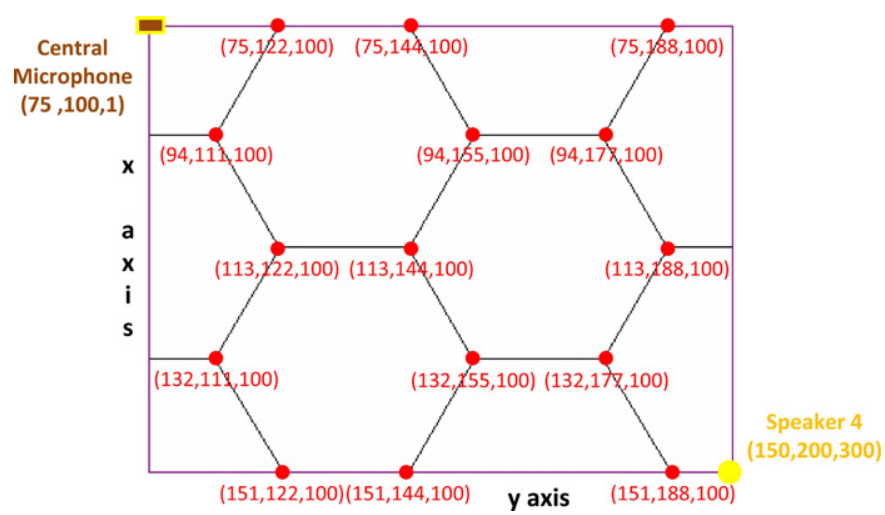

Fig. 15. Upper level test points.

The precision results for $0.02 \mathrm{~m}$ accuracy are also plotted in order to illustrate the precision distributions through the work area in Fig. 14. The precision results vary from $98.8 \%$ to $100 \%$.

On the other hand, the upper level test points were taken as the corners of hexagons as it is seen in Fig. 15.

The precision results obtained from upper level test points are illustrated in Table 3 which was constructed in a similar way as Table 2.

The precision results for $0.02 \mathrm{~m}$ accuracy at upper level are also plotted in Fig. 16. The precision results vary from $95.6 \%$ to $100 \%$.

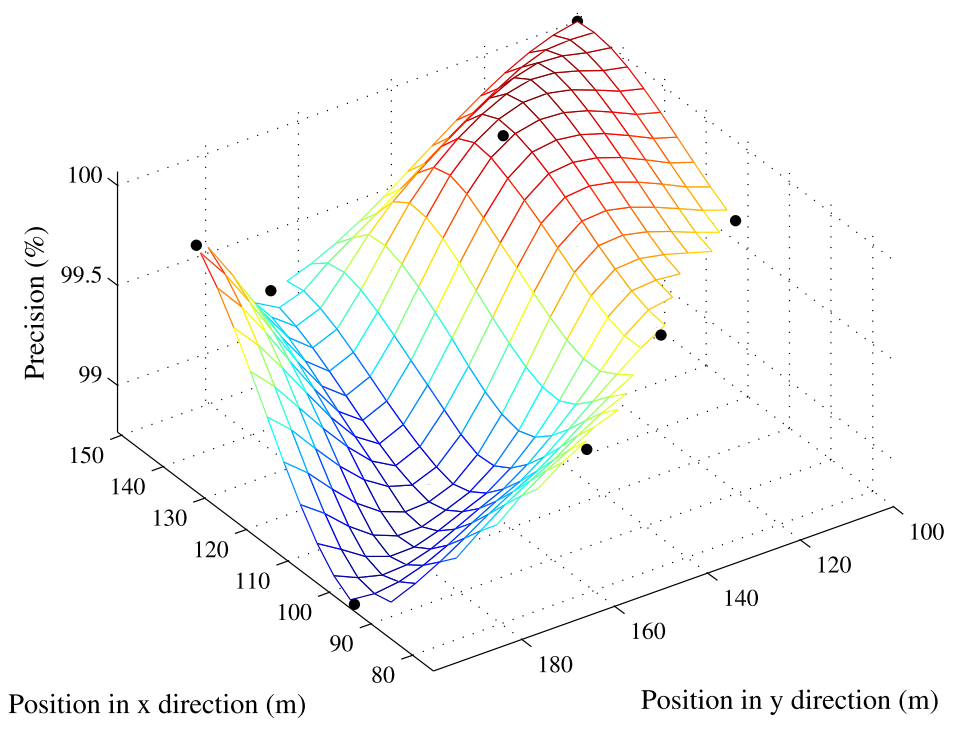

Fig. 14. Precision distribution for $0.02 \mathrm{~m}$ accuracy at the ground level. 
Table 3

Percentages of precision at upper level.

\begin{tabular}{|c|c|c|c|c|c|c|}
\hline \multirow[t]{2}{*}{ Points } & \multicolumn{6}{|l|}{ Accuracy } \\
\hline & $0.005 \mathrm{~m}$ & $0.01 \mathrm{~m}$ & $0.015 \mathrm{~m}$ & $0.02 \mathrm{~m}$ & $0.025 \mathrm{~m}$ & $0.03 \mathrm{~m}$ \\
\hline $94,111,100$ & 0 & 14.8 & 100 & 100 & 100 & 100 \\
\hline $132,111,100$ & 31.2 & 98.8 & 99.6 & 99.6 & 99.6 & 99.6 \\
\hline $75,122,100$ & 0.4 & 75.2 & 100 & 100 & 100 & 100 \\
\hline $113,122,100$ & 6.4 & 99.2 & 100 & 100 & 100 & 100 \\
\hline $151,122,100$ & 36.8 & 99.2 & 100 & 100 & 100 & 100 \\
\hline $75,144,100$ & 0 & 5.6 & 58.8 & 99.2 & 100 & 100 \\
\hline $113,144,100$ & 0.8 & 79.2 & 99.2 & 99.2 & 99.2 & 99.2 \\
\hline $151,144,100$ & 16 & 84 & 99.6 & 99.6 & 99.6 & 99.6 \\
\hline $94,155,100$ & 2 & 70.4 & 99.2 & 99.2 & 99.2 & 99.2 \\
\hline $132,155,100$ & 0 & 6.8 & 53.2 & 96 & 100 & 100 \\
\hline $94,177,100$ & 0 & 0.4 & 47.2 & 96.8 & 100 & 100 \\
\hline $132,177,100$ & 0 & 0.4 & 62.8 & 94.4 & 99.2 & 99.2 \\
\hline $75,188,100$ & 0 & 2.4 & 49.6 & 98.4 & 99.6 & 99.6 \\
\hline $113,188,100$ & 0 & 53.2 & 96.4 & 99.2 & 99.6 & 99.6 \\
\hline $151,188,100$ & 0 & 0.8 & 64.4 & 95.6 & 98.8 & 98.8 \\
\hline
\end{tabular}

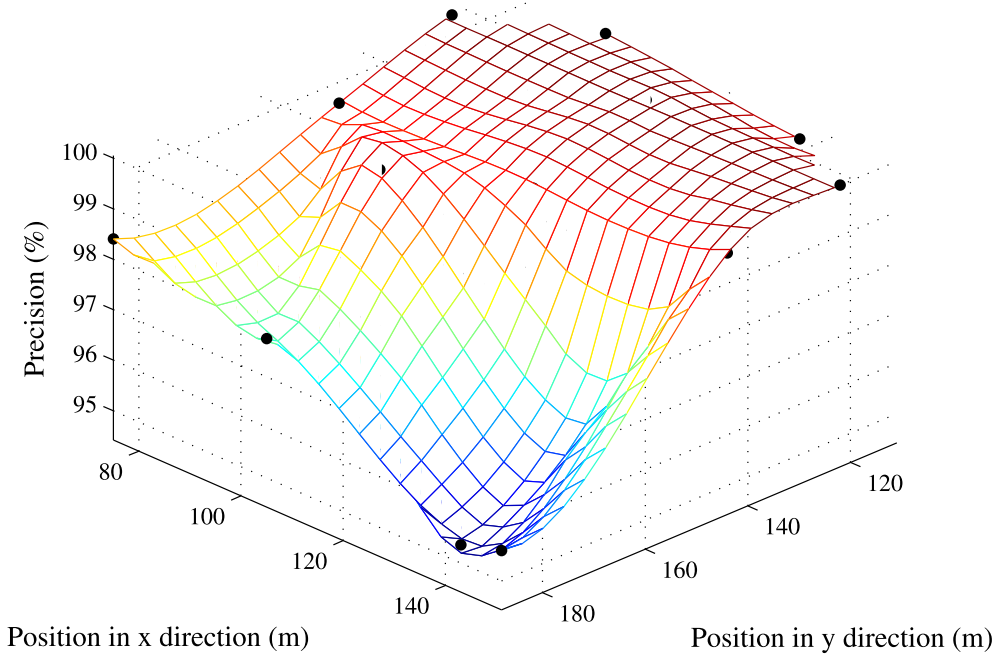

Fig. 16. Precision distribution for $0.02 \mathrm{~m}$ accuracy at the upper level.

After precision results were obtained, several conclusions can be drawn as:

- Precision decreases when the test point is moved away from the center

- Precision decreases while the test point is getting closer to speakers level in height,

- Precision results for all tests points are considerably high that the proposed system can be used for the applications that require approximately $2 \mathrm{~cm}$ accuracy with substantially precise results.

The decrease in precision in both of the cases mentioned above is related to the deterioration of the speakers' performances as the angle of the direction from the normal increases. The performances of speakers are given in [28].

After examining the precision results, the precision versus accuracy graphs were plotted in order to see the overall performance of the system.

As shown in Fig. 17, at the ground level $100 \%$ precision was achieved at $1.9 \mathrm{~cm}$ accuracy whereas at the upper level $100 \%$ precision was obtained at $2.2 \mathrm{~cm}$ accuracy.

Although the proposed system performs the computations in a central unit, it can be accepted as a privacy-oriented system, since the system does not emit any detectable signals and uses its transducer to receive the signals as it is mentioned in [17].

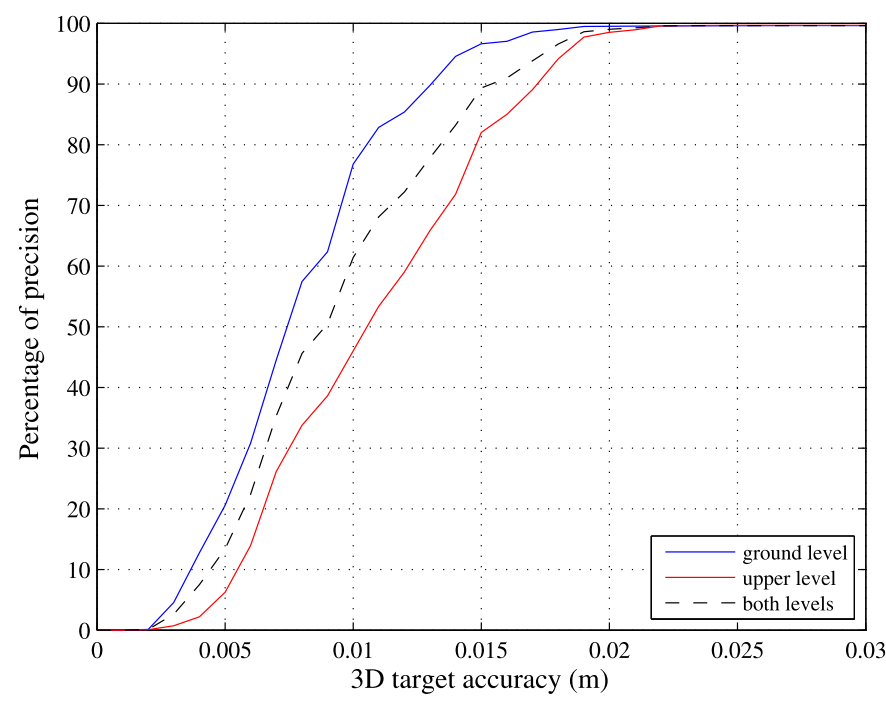

Fig. 17. Precision versus accuracy.

If a performance comparison is made among Dolphin system, 3D-LOCUS LPS and the proposed location system, it is seen that the proposed system provides location estimation better than $2 \mathrm{~cm}$ accuracy within $99 \%$ precision, while Dolphin system provides accuracy about $4.9 \mathrm{~cm}$ within $67 \%$ returned readings in synchronous 
privacy-oriented mode and 3D-LOCUS system provides $13.5 \mathrm{~mm}$ accuracy in $95 \%$ confidence level within $94.7 \%$ valid readings in private CDMA configuration. But, it is worth to mention that in the experiment with the proposed system the results were not separated into valid and non-valid outcomes as in the other systems, so none of them was rejected in order to see the general attitude of the system. Despite to the fact that the proposed system utilizes only ordinary, inexpensive equipment, it provides successful results as good as the ones offered by other systems.

\section{References}

[1] P. Daly, Navstar GPS and GLONASS: Global satellite navigation system, Electron. Commun. Eng. J. (1993) 349-357.

[2] H. Liu, H. Darabi, P. Banerjee, J. Liu, Survey of wireless indoor positioning techniques and systems, IEEE Trans. Syst. Man Cybern. C 37 (2007) 1067-1080.

[3] I. Getting, The global positioning system, IEEE Spectrum 30 (1993) 36-47.

[4] Y. Gu, A. Lo, I. Niemegeers, A survey of indoor positioning systems for wireless personal networks, IEEE Commun. Surveys Tutorials 11 (2009) 13-31.

[5] R. Want, A. Hopper, V. Falcão, J. Gibbons, The active badge location system, ACM Trans. Inf. Syst. 10 (1) (1992) 91-102.

[6] C. Lee, et al., Indoor positioning system based on incident angles of infrared emitters, in: IECON 2004, 30th Annual Conference of IEEE, vol. 3, 2-6 November 2004, Industrial Electronics Society, 2004, pp. 2218-2222.

[7] Xavier N. Fernando, Krishnan Srithar, Sun Hongbo, Kazemi-Moud Kamyar, Adaptive denoising at infrared wireless receivers, in: Bjorn F. Andresen, Gabor F. Fulop (Eds.), Infrared Technology and Applications XXIX, in: Proceedings of the SPIE, vol. 5074, 2003, pp. 199-207.

[8] P. Bahl, V.N. Padmanabhan, RADAR: An in-building RF-based user location and tracking system, Proc. IEEE INFOCOM 2 (2000) 775-784.

[9] P. Bahl, V.N. Padmanabhan, A. Balachandran, Enhancements to the RADAR user location and tracking system, Microsoft Research Technical Report, http:// research.microsoft.com/ bahl/papers/pdf/msr-tr-2000-12.pdf, 2000 (accessed April 25, 2009).

[10] L.M. Ni, Y. Liu, Y.C. Lau, A.P. Patil, LANDMARC: Indoor location sensing using active RFID, Wireless Networks 10 (6) (2004) 701-710.

[11] B. Dawes, K.-W. Chin, A comparison of deterministic and probabilistic methods for indoor localization, J. Syst. Software 84 (2011) 442-451.

[12] A. Harter, A. Hopper, P. Steggles, A. Ward, P. Webster, The anatomy of a contextaware application, Wireless Networks 8 (2) (2002) 187-197.

[13] N.B. Priyantha, A. Chakraborty, H. Balakrishnan, The Cricket location support system, in: Proceedings of the 6th Annual International Conference on Mobile Computing and Networking, 2000, pp. 32-43.

[14] N.B. Priyantha, The Cricket indoor location system, PhD thesis, Massachusetts Institute of Technology, 2005.

[15] N.B. Priyantha, A.K. Miu, H. Balakrishnan, S. Teller, The Cricket compass for context-aware mobile applications, in: Proceedings of the 7th Annual International Conference on Mobile Computing and Networking, 2001, pp. 1-14.

[16] M. Hazas, A. Ward, A high performance privacy-oriented location system, in: Proceedings of the First IEEE International Conference on Pervasive Computing and Communications, 2003, pp. 216-223.

[17] M. Hazas, A. Hopper, Broadband ultrasonic location systems for improved indoor positioning, IEEE Trans. Mobile Comput. 5 (5) (2006) 536-547.

[18] B. Cyril, T. Conners, G. Leon, S. Pradhan, An autonomous, self-assembling sensor network for indoor asset and system management, HP Laboratories Technical Report, http://www.hpl.hp.com/techreports/2003/HPL-2003-41.pdf, 2005 (accessed April 26, 2009).

[19] H. Piontek, M. Seyfferm, J. Kaiser, Improving the accuracy of ultrasound based localisation systems, Personal Ubiquitous Comput. 11 (6) (2007) 439-449.

[20] J.C. Prieto, A.R. Jiménez, J.I. Guevara, J.L. Ealo, F.A. Seco, J.O. Roa, J.X. Ramos, Subcentimeter-accuracy localization through broadband acoustic transducers, in: Proc. IEEE International Symposium on Intelligent Signal Processing, 2007, p. 16.

[21] J.C. Prieto, A.R. Jiménez, J.I. Guevara, J.L. Ealo, F.A. Seco, J.O. Roa, F. Ramos, Performance evaluation of 3D-LOCUS advanced acoustic LPS, IEEE Trans. Instrum. Meas. 58 (2009) 2385-2395.

[22] M. Alloulah, M. Hazas, An efficient CDMA core for indoor acoustic position sensing, in: Proceedings of IPIN 2010: International Conference on Indoor Positioning and Indoor Navigation, Zurich, Switzerland, September 2010.

[23] F.J. Alvarez, T. Aguilera, J.A. Fernandez, J.A. Moreno, A. Gordillo, Analysis of the performance of an ultrasonic local positioning system based on the emission of Kasami codes, in: Proceedings of IPIN 2010: International Conference on Indoor Positioning and Indoor Navigation, Zurich, Switzerland, September 2010.

[24] H. Schweinzer, M. Syafrudin, LOSNUS: An ultrasonic system enabling high accuracy and secure TDoA locating of numerous devices, in: Proceedings of IPIN
2010: International Conference on Indoor Positioning and Indoor Navigation, Zurich, Switzerland, September 2010.

[25] K. Mizutani, T. Ito, M. Sugimoto, H. Hashizume, Fast and accurate ultrasonic 3D localization using the TSaT-MUSIC algorithm, in: Proceedings of IPIN 2010: International Conference on Indoor Positioning and Indoor Navigation, Zurich, Switzerland, September 2010.

[26] S. Nakamura, T. Sato, M. Sugimoto, H. Hashizume, An accurate technique for simultaneous measurement of 3D position and velocity of a moving object using a single ultrasonic receiver unit, in: Proceedings of IPIN 2010: International Conference on Indoor Positioning and Indoor Navigation, Zurich, Switzerland, September 2010 .

[27] J.M. Martin, A.R. Jimenez, F. Seco, L. Calderon, J.L. Pons, R. Ceres, Estimating the 3D-position from time delay data of US-waves: experimental analysis and a new processing algorithm, Sens. Actuators A 101 (3) (2002) 311-321.

[28] C. Sertatil, Acoustic indoor localization employing code division multiple access, M.S. thesis, Izmir Institute of Technology, 2010.

Cem Sertatıl was born in İzmir, Turkey, in 1984. He received his B.S. degree in Electrical and Electronics Engineering from Bilkent University, Ankara, Turkey, in 2007. He received his M.S. degree in Electronics and Communication Engineering from İzmir Institute of Technology, İzmir, Turkey, in 2010. He had been an exchange student in Polytech Grenoble, Universite Joseph Fourier, Grenoble, France, through the fall semester of 2008-2009. His research interests include digital communication, digital signal processing, dynamical systems and localization. In April 2011, he joined Vestel White Goods Co., Manisa, Turkey, where he has been an engineer in R\&D Department of Washing Machine Plant. Additionally, he has been studying MBA in İzmir University of Economics, İzmir, Turkey, since September 2011.

Mustafa A. Altınkaya was born in Rize, Turkey, in 1965. He received the B.S., M.S. and Ph.D. degrees in electrical and electronics engineering from Boğaziçi University, Istanbul, Turkey, in 1987, 1990 and 1996, respectively.

From 1989 to 1990 he was a research associate in TUBITAK Marmara Research Center, Kocaeli, Turkey. From 1991 to 1996 he was a research and teaching assistant in Boğaziçi University. He worked as a part-time instructor in Air Force Academy, Naval Academy and Boğaziçi University during periods from 1995 to 1998. In 1998 he joined the Electrical and Electronics Engineering Department at Izmir Institute of Technology where he has been an assistant professor.

He is the author of more than 20 publications. His research interests are mainly on statistical signal processing and its applications in telecommunications, source separation, structural dynamic systems, and cheminformatics.

Kosai Raoof is currently a full professor at the ENSIM Engineering College, University of Maine, Le Mans. He joined the signal processing and transducers group of LAUM Laboratory in 2011. He obtained his M.Sc. and Ph.D. from Grenoble University in 1990 and 1993 respectively; in 1998 he obtained the Habilitation à Diriger Des Recherches Degree (HDR). He was invited to join Laboratoire des Images et Signaux (LIS) in 1999, to participate in the founding of telecommunication research group. His research interest was first focalized on advanced MIMO systems and joint CDMA synchronization; he studied and introduced polarized diversity in MIMO systems. In 2007 he joined GIPSA-LAB to continue his research on Smart Sensor Networks and cooperative MIMO antenna systems. He is a referee for many international journals and conferences in the field of telecommunications and signal processing. He published more than 70 papers and project reports in the field of signal processing. He was granted for European IST Program QTPACK and RNRT French research program ASTURIES in telecommunications in 2004. He supervised more than $20 \mathrm{Ph} . \mathrm{D}$. and M.Sc. students in different fields of applied signal processing and telecommunications. He is a chief editor for a new publishing series of advanced telecommunication systems, the first book 'Advanced MIMO Systems' and the second book 'Advanced Cognitive Radio Network' were published in summer 2009 and in 2011 respectively. He is also the chief editor of 'Wireless Sensor Networks' Journal first published in 2008. His recent interest is localization for smart sensor networks and distributed agents for intelligent spaces. 